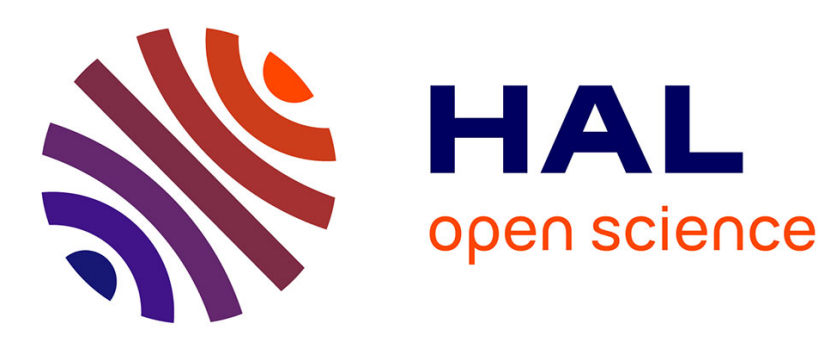

\title{
Synchronized Swept-Sine: Theory, Application, and Implementation
}

\author{
Antonin Novak, Laurent Simon, Pierrick Lotton
}

\section{To cite this version:}

Antonin Novak, Laurent Simon, Pierrick Lotton. Synchronized Swept-Sine: Theory, Application, and Implementation. Journal of the Audio Engineering Society, 2015, 63 (10), pp.786-798. 10.17743/jaes.2015.0071 . hal-02504321

\section{HAL Id: hal-02504321 \\ https://hal.science/hal-02504321}

Submitted on 10 Mar 2020

HAL is a multi-disciplinary open access archive for the deposit and dissemination of scientific research documents, whether they are published or not. The documents may come from teaching and research institutions in France or abroad, or from public or private research centers.
L'archive ouverte pluridisciplinaire HAL, est destinée au dépôt et à la diffusion de documents scientifiques de niveau recherche, publiés ou non, émanant des établissements d'enseignement et de recherche français ou étrangers, des laboratoires publics ou privés. 


\title{
Synchronized Swept-Sine: Theory, Application and Implementation
}

\author{
Antonin Novak*, Laurent Simon, Pierrick Lotton \\ Laboratoire d'Acoustique de I'Université du Mans, LAUM - UMR 6613 CNRS, Le Mans Université, Avenue Olivier \\ Messiaen, 72085 LE MANS CEDEX 9, France \\ *antonin.novak@univ-lemans.fr \\ *https://ant-novak. com
}

\begin{abstract}
Exponential, or sometimes called logarithmic, swept-sine signal is very often used to analyze nonlinear audio systems. In this paper, the theory of exponential swept-sine measurements of nonlinear systems is reexamined. The synchronization procedure necessary for a proper analysis of higher harmonics is detailed leading to an improvement of the formula for the exponential swept-sine signal generation. Moreover, an analytical expression of spectra of the swept-sine signal is derived and used in the deconvolution of the impulse response. A Matlab code for generation of the synchronized swept-sine, deconvolution, and separation of the impulse responses is given with discussion of some application issues and an illustrative example of harmonic analysis of current distortion of a woofer is provided.
\end{abstract}

The archived file is not the final published version of the article A. Novak, P. Lotton \& L. Simon (2015), "Synchronized

Swept-Sine: Theory, Application, and Implementation", Journal of the Audio Engineering Society. Vol. 63(10), pp. 786-798.

The definitive publisher-authenticated version is available online at https://doi.org/10.17743/jaes.2015.0071, Readers must contact the publisher for reprint or permission to use the material in any form. 


\section{INTRODUCTION}

A swept-sine, also called a chirp, is a widely used signal for characterizing a frequency response of a linear system under test. A technique for analysis of nonlinear system (NLS) based on an exponential swept-sine signal has been first presented by Farina in 2000. This technique enables a separation of impulse responses for each harmonic distortion order [1]. Later, Farina called this technique the "nonlinear convolution" [2]. It is worth noting that the idea of separation of harmonic products when using the swept-sine signals was mentioned earlier in [3, 4].

Since 2000, the method has been used in many applications, namely in fields of audio and acoustics, e.g. for Room Impulse Response (RIR) measurements [5, 6], for measurement of Head-Related Transfer Functions (HRTF) and Head-Related Impulse Responses (HRIR) [7, 8], in nonlinear acoustics [9, 10], and in many other applications in acoustics $[11,12,13,14]$.

The need to synchronize the exponential swept-sine signal for purpose of nonlinear system identification has been first presented in [15] and the mathematical background for the identification of nonlinear systems using the exponential swept-sine signal has been then given in [16]. The importance of the synchronization of the swept-sine signal for identification of a cascade of Hammerstein systems has been also pointed out in [17]. It has also been shown in [18] that the method based on the exponential swept-sine signal can be used to identify two nonlinear systems in series.

In this paper, we reformulate the exponential swept-sine method. After a short review of the method presented in Sec. 2 we derive the form of the exponential swept-sine signal suitable for the estimation of so-called Higher Harmonic Frequency Responses (HHFRs) in Sec. 3. This form differs from the one usually used with the exponential sweptsine methods and we focus on consequences of different definitions. Next, the analytical formula for the spectra of the synchronized swept-sine signal is derived in Appendix and used for the deconvolution in Sec. 4. In Sec. 5, the separation of higher harmonic impulse responses is described and the advantage of using the analytical formula in deconvolution procedure is discussed. The most important equations for the synchronized swept-sine method are then summarized in Sec. 6. Finally, we provide an illustrative example in Sec. 7 in which we analyze higher harmonics of current distortion of a woofer using both the synchronized and the non-synchronized swept-sine signals and we compare the results with a sine-based measurement. Moreover, in Sec. A.2 a Matlab code for all the important steps of the method is provided and some implementation issues are discussed.

\section{Brief Review Of the Swept-Sine Method}

Regarding the basics of NLS theory, when a sine-wave is used as an input signal higher harmonics appear at the output of the NLS as multiples of the input driving frequency. The amplitude and phase of all the higher harmonics may be furthermore frequency dependent. In this section, we briefly recall the main steps of the exponential sweptsine method to separate the frequency dependent higher harmonics by using a single swept-sine measurement.

The exponential swept-sine method can be summarized in the following steps:

- generation of the exponential swept-sine input signal

- acquisition of the response of the nonlinear system under test to the exponential swept-sine signal

- impulse response deconvolution

- separation of the higher harmonic impulse responses $h_{n}(t)$ and estimation of so-called Higher Harmonic Frequency Responses (HHFRs) $H_{n}(f)$ (Fourier transform of the higher harmonic impulse responses)

\subsection{Exponential Swept-Sine Signal Generation}

The exponential swept-sine signal can be generated either directly in the time domain or indirectly in the frequency domain as discussed in [19]. The generation in time domain can be realized using a direct-implementation method, 
also discussed in [19], or using a mathematical formula given by Farina in [1] as

$$
x(t)=\sin \left\{\frac{2 \pi f_{1} T}{\ln \left(f_{2} / f_{1}\right)}\left[\exp \left(\frac{t}{T} \ln \left(\frac{f_{2}}{f_{1}}\right)\right)-1\right]\right\},
$$

where $f_{1}$ is the initial frequency, $f_{2}$ is the final frequency and $T$ is the time duration of the swept-sine signal.

It has been shown in [16] that the swept-sine signal must be generated in a slightly different way in order to be able to estimate both amplitude and phase of HHRFs properly. The modified signal is called synchronized swept-sine. It is generated according to [16] as

$$
x(t)=\sin \left\{2 \pi f_{1} L\left[\exp \left(\frac{t}{L}\right)-1\right]\right\},
$$

$L$ being the rate of frequency increase defined as

$$
L=\frac{1}{f_{1}} \text { round }\left[\frac{\hat{T} f_{1}}{\ln \left(\frac{f_{2}}{f_{1}}\right)}\right]
$$

where $\hat{T}$ is an approximate time length of the signal $x(t)$ and round represents rounding towards nearest integer. According to [16], the rounding operation is necessary to avoid an incorrect phase estimation of HHRFs. The phase synchronization of HHFRs has been successfully used in several works dealing with nonlinear system identification in acoustics [8, 20].

\subsection{Impulse Response Deconvolution}

The exponential swept-sine signal $x(t)$ is then used as the input to the nonlinear system under test and the output $y(t)$ signal is acquired. To obtain the impulse response $h(t)$ of the nonlinear system under test, we deconvolve the output signal $y(t)$ with the original swept-sine signal $x(t)$.

The deconvolution can be made either in time or in frequency domain. The classical approach consists in deconvolution by division in frequency domain as

$$
h(t)=\mathcal{F}^{-1}\left[\frac{\mathcal{F}[y(t)]}{\mathcal{F}[x(t)]}\right],
$$

where $x(t)$ is the swept-sine signal, $y(t)$ is the (system) response to $x(t)$ and $\mathcal{F}$ and $\mathcal{F}^{-1}$ are direct and inverse Fourier transforms respectively. A regularization function should be added in order to avoid division by zero problems since $\mathcal{F}[x(t)]$ approach zero below $f_{1}$ and above $f_{2}[19,21]$.

The approach introduced by Farina [1] consists in using a so-called inverse filter $\tilde{x}(t)$ defined in such a way that, when convolved with the swept-sine signal $x(t)$, we get the Dirac delta function $\delta(t)$

$$
x(t) * \tilde{x}(t)=\delta(t)
$$

According to [21], the inverse filter is a time-reversal of the swept-sine signal $x(t)$ with $+3 \mathrm{~dB} /$ octave slope. The deconvolution can then be realized either in the time domain

$$
h(t)=y(t) * \tilde{x}(t),
$$

or in frequency domain

$$
h(t)=\mathcal{F}^{-1}[\mathcal{F}[y(t)] \mathcal{F}[\tilde{x}(t)]] .
$$




\subsection{Higher Harmonic Impulse Responses Separation}

The deconvolution process results in the impulse response $h(t)$ which is a summation of time-shifted higher harmonic impulse responses $h_{n}(t)[1,16]$. They can be easily separated by windowing in the time domain as described in $[1,19]$. Finally, they can be Fourier transformed to calculate the HHFRs as

$$
H_{n}(f)=\mathcal{F}\left[h_{n}(t)\right]
$$

The magnitude plot of $H_{n}(f)$ in $\mathrm{dB}$ is the most common representation since it shows the amount of distortion of all the harmonics as a function of frequency [1].

\section{Synchronized Swept-Sine Signal Generation}

In this section we focus on the generation of the exponential swept-sine signal. We give the mathematical background to the choice of the form of the signal and we explain why the synchronization of the swept-sine signal is necessary for a correct estimation of the HHFRs $H_{n}(f)$.

\subsection{Conditioning the Swept-Sine Signal}

The properties of the exponential swept-sine signal generated either using (1) or (2) have been studied in [1] and [16] respectively. Instead of discussing the properties of a given exponential swept-sine signal, we can formulate the problem in another way and rise the following question: What kind of swept-sine signal can be used for the estimation of the HHFRs $H_{n}(f)$ ?

To answer this question, we start with a general definition of a swept-sine signal $x(t)$ with an unknown phase $\varphi(t)$

$$
x(t)=\sin [\varphi(t)],
$$

and we consider the response $y(t)$ of the NLS to such a signal as a sum of higher harmonics as

$$
y(t)=\sum_{n=1}^{\infty} \sin [n \varphi(t)] * h_{n}(t) .
$$

Considering that the response $y(t)$ of the NLS to the swept-sine signal $x(t)$ is simply a convolution of the latter with an impulse response $h(t)$

$$
y(t)=x(t) * h(t),
$$

we want the impulse response to be a sum of the time-delayed higher harmonic impulse responses $h_{n}(t)$ as

$$
h(t)=\sum_{n=1}^{\infty} h_{n}\left(t+\Delta t_{n}\right)
$$

so that they can be easily separated from each other. From (9), (11), and (12) we can write

$$
y(t)=\sin [\varphi(t)] * \sum_{n=1}^{\infty} h_{n}\left(t+\Delta t_{n}\right) .
$$

Comparing the output $y(t)$ from (10) and (13) we get

$$
\sum_{n=1}^{\infty} \sin [n \varphi(t)] * h_{n}(t)=\sin [\varphi(t)] * \sum_{n=1}^{\infty} h_{n}\left(t+\Delta t_{n}\right),
$$

and putting the input signal $\sin [\varphi(t)]$ inside the convolution sum, we can write

$$
\sum_{n=1}^{\infty} \sin [n \varphi(t)] * h_{n}(t)=\sum_{n=1}^{\infty} \sin [\varphi(t)] * h_{n}\left(t+\Delta t_{n}\right) .
$$


Both sums of Eq. (15) are equal if the convolution terms inside the sum are equal as well. Expressing the inside convolution equality leads to

$$
\begin{aligned}
& \int_{-\infty}^{\infty} \sin [n \varphi(u)] h_{n}(t-u) d u= \\
& \int_{-\infty}^{\infty} \sin [\varphi(v)] h_{n}\left(t+\Delta t_{n}-v\right) d v .
\end{aligned}
$$

Substituting $v=\tau+\Delta t_{n}$, we get

$$
\begin{aligned}
\int_{-\infty}^{\infty} \sin [n \varphi(u)] & h_{n}(t-u) d u= \\
& \int_{-\infty}^{\infty} \sin \left[\varphi\left(\tau+\Delta t_{n}\right)\right] h_{n}(t-\tau) d \tau
\end{aligned}
$$

which can be solved by imposing

$$
\sin [n \varphi(t)]=\sin \left[\varphi\left(t+\Delta t_{n}\right)\right] .
$$

In other words, the signal we are looking for must have the following property: shifting the signal in time by $\Delta t_{n}$ is equivalent to generating the same signal with $n$-times higher phase.

\subsection{Synchronizing the Swept-Sine Signal}

The condition given in (18) can be solved using

$$
n \varphi(t)=\varphi\left(t+\Delta t_{n}\right)+2 \pi l, \quad \forall l \in \mathbb{Z}
$$

This equation has a form similar to that of a Delayed Differential Equation [22], which may have an oscillatory solution leading to a phase / frequency modulated signal, or to an exponential solution leading to a swept-sine signal. Focusing on the exponential solution, we consider a trial solution in form of an exponential function as

$$
\varphi(t)=A \exp (\alpha t)
$$

and inserting the trial solution back to (19), we find that

$$
\Delta t_{n}=\frac{\ln \left(n-\frac{2 \pi l}{A \exp (\alpha t)}\right)}{\alpha} .
$$

Since we want $\Delta t_{n}$ to be independent on time $t$, we must impose $l=0$ and we then get

$$
\alpha=\frac{\ln (n)}{\Delta t_{n}} .
$$

To summarize this part, we prove that the signal $x(t)$ has a form of

$$
x(t)=\sin [A \exp (\alpha t)],
$$

where the coefficient $A$ acts as a rate of sweeping change and where the coefficient $\alpha$ is still unknown. To find the relation between these two coefficients and the parameters of the swept-sine, such as the initial and the final frequencies $f_{1}$ and $f_{2}$ and the time duration $T$, the following steps must be completed. 


\subsection{Initial and Final Frequencies}

First, we define the instantaneous frequency of the signal $x(t)$ from (20) as

$$
f_{i}(t)=\frac{1}{2 \pi} \frac{d \varphi(t)}{d t}=\frac{A \alpha}{2 \pi} \exp (\alpha t) .
$$

At initial time $t=0$, the initial frequency is denoted $f_{i}(0)=f_{1}$, leading to

$$
\alpha=\frac{2 \pi f_{1}}{A} .
$$

At final time $t=T$, the final frequency is denoted $f_{i}(T)=f_{2}$, leading to

$$
f_{1} \exp \left(\frac{2 \pi f_{1}}{A} T\right)=f_{2},
$$

and thus

$$
A=\frac{2 \pi f_{1} T}{\ln \left(\frac{f_{2}}{f_{1}}\right)} .
$$

So, the formula for the synchronized swept-sine signal can be written as

$$
x(t)=\sin \left[\frac{2 \pi f_{1} T}{\ln \left(f_{2} / f_{1}\right)} \exp \left(\frac{t}{T} \ln \left(\frac{f_{2}}{f_{1}}\right)\right)\right] .
$$

Note that this formula is almost equivalent to the one given in (1) except for the "-1" term at the end of (1) that is missing in (28).

\subsection{Initial Phase}

When examining the missing term " -1 " in (28) in comparison with (1), we first note, that the term " -1 " in (1) ensures having the initial phase to be zero, which is not the case in (28), where the initial phase is given by the term

$$
\varphi(0)=\frac{2 \pi f_{1} T}{\ln \left(f_{2} / f_{1}\right)} .
$$

However, fixing the phase to be zero by adding the term "-1" leads to a different definition of the swept-sine signal that does not obey the condition given in (18) and leads to a non-synchronized version of the exponential swept-sine signal. This situation is depicted in Fig. 1a, where the exponential swept-sine signal generated using (1) is not synchronized with its time-shifted second-harmonic copy.

The signal depicted in Fig. 1b, generated using (28), is obviously well synchronized, but the initial phase does not start at origin. To overcome the initial phase problem while keeping the synchronization correct, we fix the phase to zero at $t=0$, i.e. $\varphi(0)=2 \pi k$, that is according to $(29)$

$$
\frac{2 \pi f_{1} T}{\ln \left(f_{2} / f_{1}\right)}=2 \pi k
$$

and we get

$$
k=\frac{f_{1} T}{\ln \left(f_{2} / f_{1}\right)},
$$

where $k \in \mathbb{Z}_{\neq 0}$. Note that, since $k$ is an integer, the time $T$ and the frequencies $f_{1}$ and $f_{2}$ have to be dependent. In practice, we can define the start frequency $f_{1}$, the stop frequency $f_{2}$ and an approximative duration $\tilde{T}$ of the swept-sine signal. The integer $k$ can then be calculated as

$$
k=\operatorname{round}\left[\frac{f_{1}}{\ln \left(\frac{f_{2}}{f_{1}}\right)} \tilde{T}\right],
$$




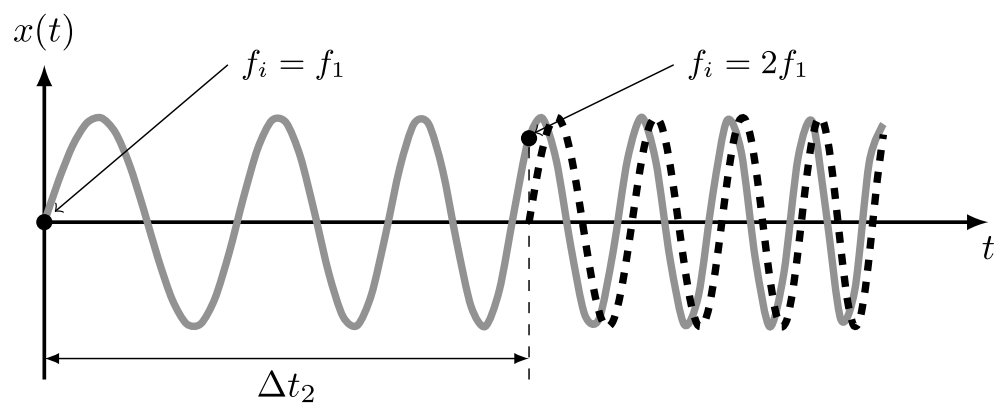

(a) non-synchronized swept-sine signal

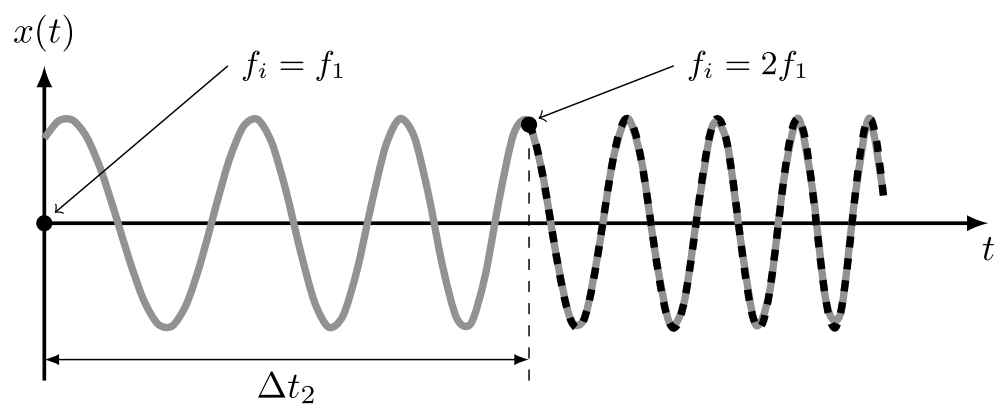

(b) synchronized swept-sine signal starting with a non-zero phase

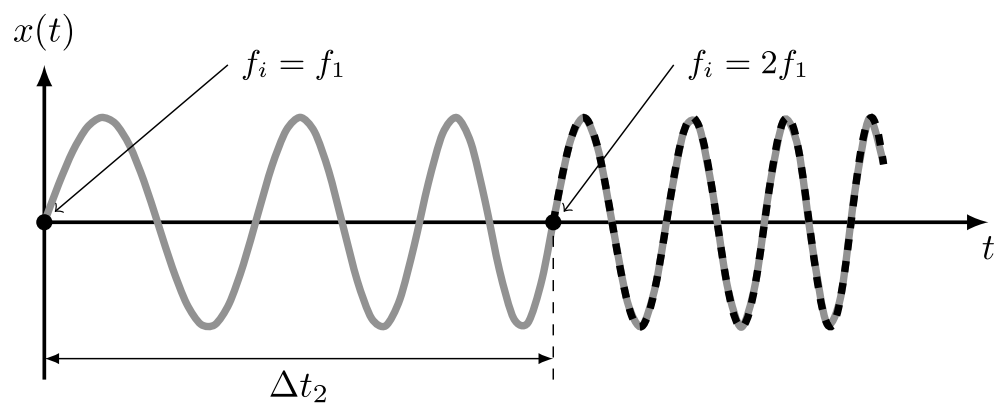

(c) synchronized swept-sine signal starting with a zero phase

Figure 1. Exponential swept-sine signal generated using three different equations: a) equation (1); b) equation $(28)$; c) equation (33). The signal $x(t)$ is plotted with gray solid line, the time-shifted second harmonic is plotted with black dashed line.

the correct duration $T$ being then derived from (31).

The synchronized swept signal with fixed initial phase is thus defined as

$$
x(t)=\sin \left[2 \pi f_{1} L \exp \left(\frac{t}{L}\right)\right],
$$

where $L=1 / \alpha=k / f_{1}$ is used in accordance with (3). Note that choosing $f_{1}>f_{2}$ would lead to a negative $k$ and to a swept-sine signal with decreasing frequency. For the sake of clarity we consider here $f_{2}>f_{1}$.

The signal depicted in Fig. 1c, generated using (33), has a zero initial phase and obeys the condition given in (18). Note also that the signal generated using (2) is equivalent to the one generated by (33) since the presence of the term " $-1 "$ in (2) is useless as $f_{1} L$ is an integer and can be omitted from the equation. 


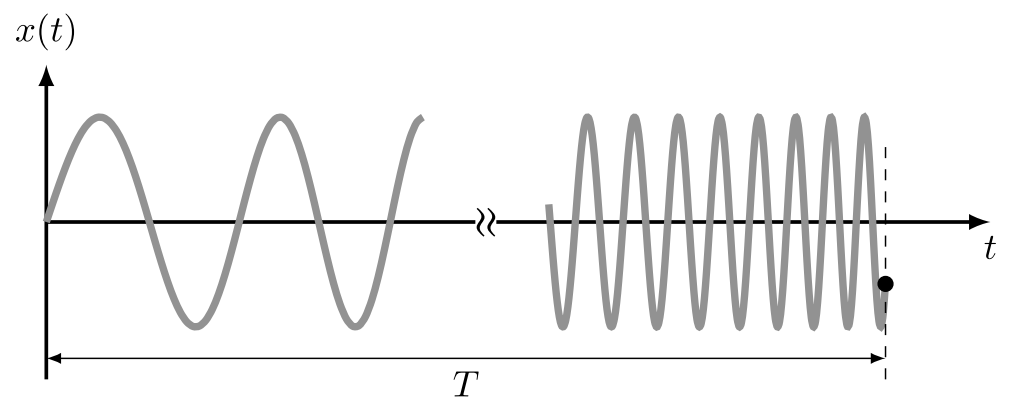

(a) a non-zero final phase at time $T ; x(t=T) \neq 0$

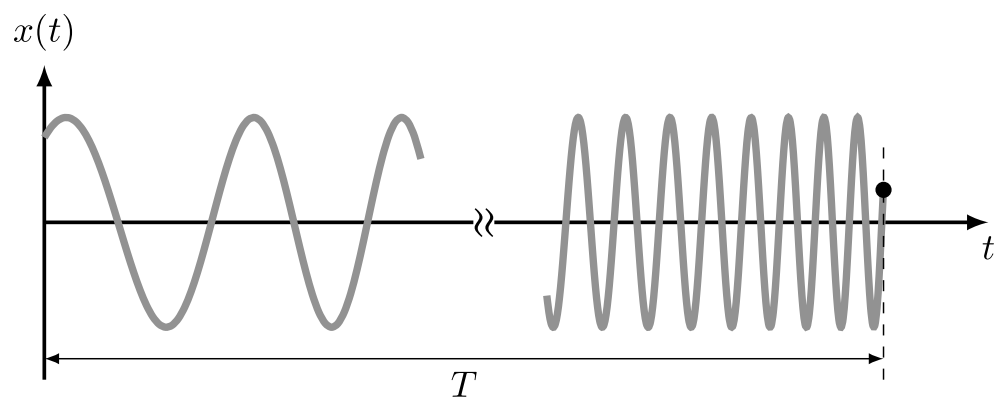

(b) a non-zero final phase at time $T ; x(t=T) \neq 0$

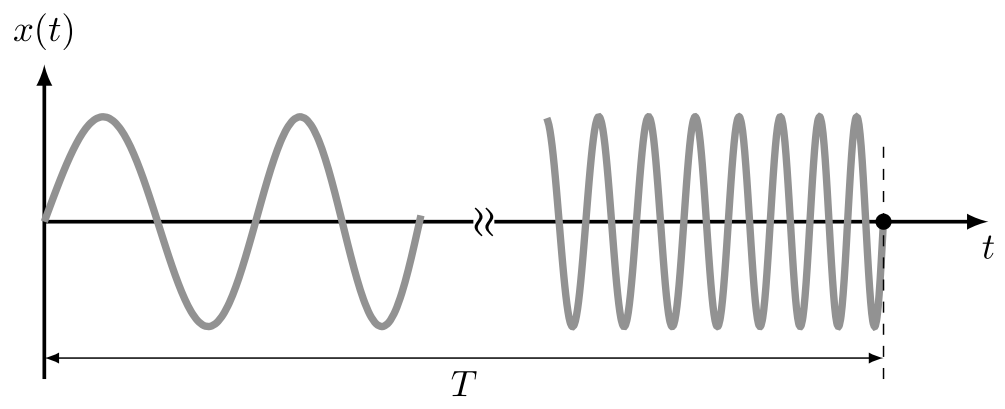

(c) a zero final phase at time $T ; x(t=T)=0$

Figure 2. The beginning and the end of the exponential swept-sine signal generated using three different equations: a) equation (1); b) equation (28); c) equation (33). Ending points are highlighted with a black circle.

\subsection{Final Phase}

The synchronized swept-sine with fixed initial phase defined in (33) has another interesting property concerning the final phase of the signal. If the final frequency $f_{2}$ is chosen as an integer multiple of the initial frequency $f_{1}$, the phase of the exponential signal $x(t)$ from (33) is zero at the time instant corresponding to the instantaneous frequency $f_{2}$. Expressed in other words, the final phase at time $T$ is also zero.

The behavior of the exponential swept-sine signals $x(t)$ near time $T$ is depicted in Fig. 2. The swept-sine signal generated using (1), depicted in Fig. 2a, exhibits a non-zero final phase at time $T$. As discussed in [21], the signal generated by this way should be cut manually at the latest zero-crossing before its abrupt termination. The synchronized swept-sine with a non-fixed initial phase generated using (28) exhibits a non-zero final phase at time $T$. The final phase is, this time, an integer multiple of the initial phase. Finally, the synchronized swept-sine with fixed initial phase generated using (33) has a zero final phase. Using this approach, we can avoid the manual cut of the tail of the swept-sine signal. 


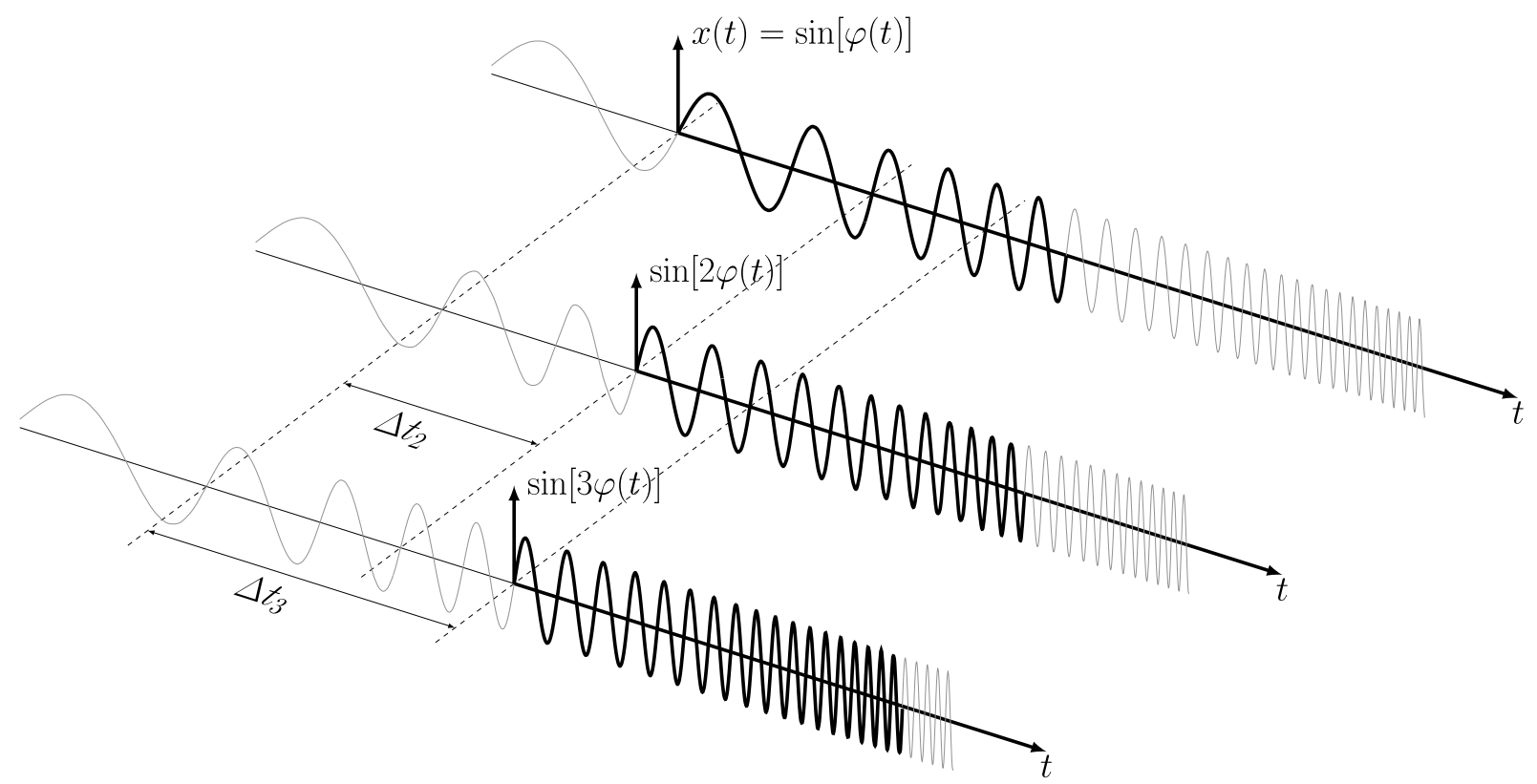

Figure 3. Delaying the Synchronized swept-sine signal $x(t)=\sin [\varphi(t)]$ by $\Delta t_{2}$ and by $\Delta t_{3}$ is equivalent to generating the second and third harmonic $\sin [2 \varphi(t)]$ and $\sin [3 \varphi(t)]$ of the Synchronized swept-sine signal $x(t)$.

\subsection{Time Delays vs. Instantaneous Frequency}

The synchronized swept-sine signal generated using (33) has the following property, due to the condition given in (18). The time-shifted synchronized swept-sine signal by time delay $\Delta t_{n}$ is equivalent to the $\mathrm{n}$-th higher harmonic copy of the signal $x(t)$ itself. The $\mathrm{n}$-th harmonic copy has a phase value, and thus also an instantaneous frequency, $n$-times higher than the one of the original synchronized swept-sine signal $x(t)$. This property is depicted in Fig. 3.

The time delays $\Delta t_{n}$ can be derived from (22) as

$$
\Delta t_{n}=L \ln (n) .
$$

Similarly, the instantaneous frequency $f_{i}(t)$ can be derived from (24) as

$$
f_{i}(t)=f_{1} \exp \left(\frac{t}{L}\right) \text {. }
$$

According to the condition given in (18) we can find a simple relation between the instantaneous frequency $f_{i}(t)$ and the time delays $\Delta t_{n}$. The time delays $\Delta t_{n}$ indicate the time instants corresponding to the instantaneous frequency (and phase) n-times higher than the initial frequency (and phase), which can be written mathematically as

$$
f_{i}\left(\Delta t_{n}\right)=n f_{1} .
$$

Moreover, thanks to the synchronization of the swept-sine and fixed initial phase, we can write

$$
x\left(\Delta t_{n}\right)=0 .
$$

Both properties are depicted together in Fig. 4.

\section{IMPULSE RESPONSE DECONVOLUTION}

The previous section discussed the generation of the synchronized swept-sine signal $x(t)$ that is used as the input to the nonlinear system under test. After the measurement, the output signal $y(t)$ is acquired and the impulse 


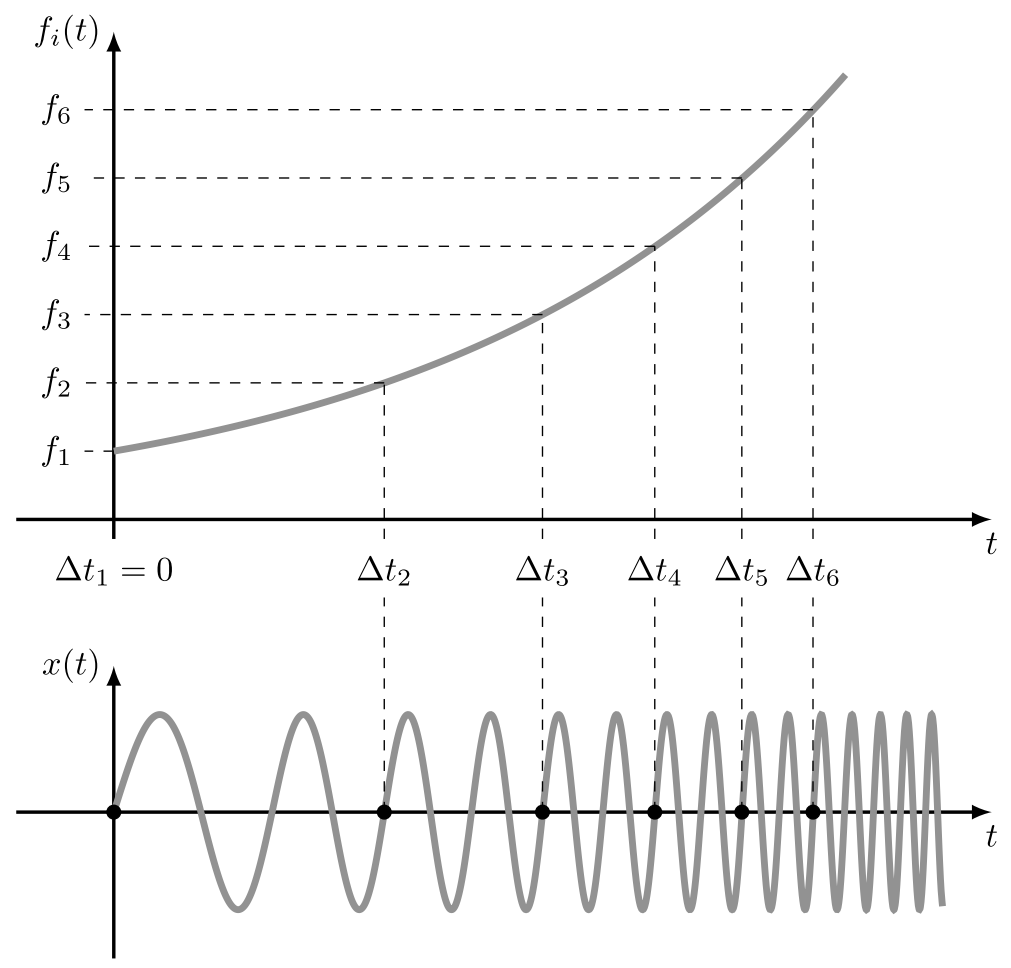

Figure 4. Synchronized swept-sine $x(t)$ (below) and the associated instantaneous frequency $f_{i}(t)$.

response consisting of time-delayed higher harmonic impulse responses can be deconvolved from the output signal $y(t)$. The impulse response deconvolution is usually performed using (4) or (7).

\subsection{Analytical Deconvolution}

Another way to deconvolve the impulse response $h(t)$ is to use an analytical expression of the inverse filter in the frequency domain $\tilde{X}(f)$ and then compute the deconvolution directly in the frequency domain as

$$
\left.h(t)=\mathcal{F}^{-1}[\mathcal{F}[y(t)] \tilde{X}(f)]\right] .
$$

In order to derive the Fourier transform of the synchronized swept-sine signal $X(f)$, we use the analytical swept-sine signal $z(t)$ defined as

$$
z(t)=\exp [j \varphi(t)]
$$

that, in relation with the swept-sine signal $x(t)$ from Eq. (33), is expressed as

$$
z(t)=x_{c}(t)+j x(t)
$$

where $x_{c}(t)=-\mathcal{H}[x(t)]$ is the cosine version of the synchronized swept-sine defined as the negative Hilbert transform of $x(t)$.

The mathematical derivation of the Fourier transform of the analytical signal $z(t)$ is provided in Appendix A.1 and results in

$$
Z(f)=\sqrt{\frac{L}{f}} \exp \left\{j 2 \pi f L\left[1-\ln \left(\frac{f}{f_{1}}\right)\right]+j \frac{\pi}{4}\right\},
$$

from which the Fourier transform of the synchronized swept-sine signal $X(f)$ can be expressed for positive frequencies 


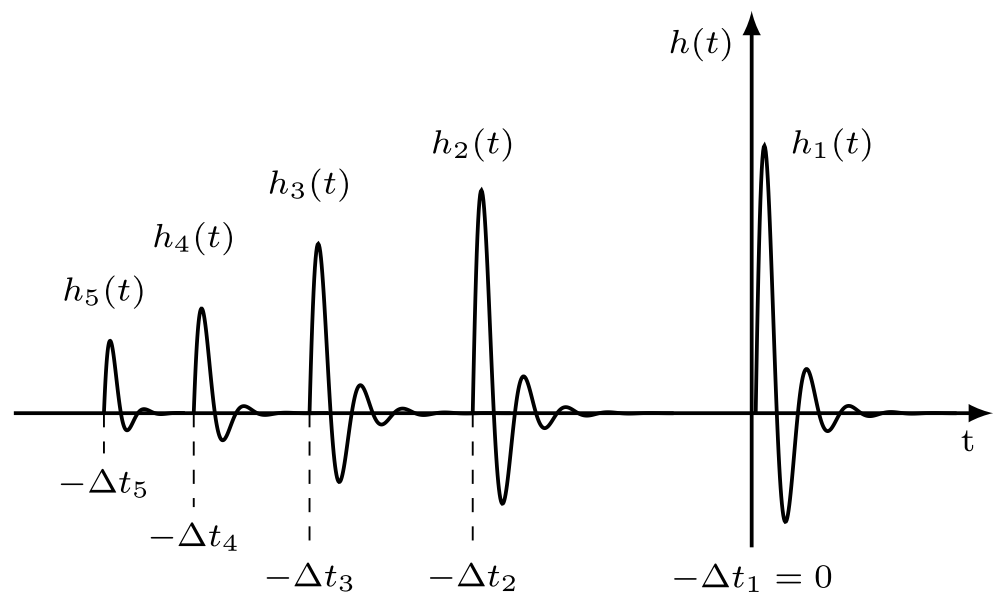

Figure 5. Result of the deconvolution process resulting in a sum of time-shifted higher harmonic impulse responses $h_{n}(t)$

$f$ as

$$
X(f)=\frac{1}{2} \sqrt{\frac{L}{f}} \exp \left\{j 2 \pi f L\left[1-\ln \left(\frac{f}{f_{1}}\right)\right]-j \frac{\pi}{4}\right\},
$$

and the Fourier transform of inverse filter $\tilde{X}(f)$ as

$$
\tilde{X}(f)=2 \sqrt{\frac{f}{L}} \exp \left\{-j 2 \pi f L\left[1-\ln \left(\frac{f}{f_{1}}\right)\right]+j \frac{\pi}{4}\right\} .
$$

\subsection{Group Delay}

Lastly, the spectra $X(f)$ from Eq. (42) has a phase such that

$$
\phi(f)=2 \pi f L\left[1-\ln \left(\frac{f}{f_{1}}\right)\right]-\frac{\pi}{4},
$$

from which the group delay can be derived according to its definition as

$$
\tau_{g}(f)=-\frac{1}{2 \pi} \frac{d \phi(f)}{d f}=L \ln \left(\frac{f}{f_{1}}\right) .
$$

Note, that the group delay from Eq. (45) is an inverse function to the instantaneous frequency $f_{i}(t)$ defined in Eq. (35). Because of this property, the signal $x(t)$ is an asymptotic signal [23, 24$]$.

\section{HIGHER HARMONICS SEPARATION}

The deconvolution process defined in Eq. (38) results in impulse response $h(t)$ which is according to Eq. (12 ) a sum of time-shifted higher harmonic impulse responses $h_{n}(t)$. A schematic example of the impulse response $h(t)$ is depicted in Fig. 5 .

The higher harmonic impulse responses $h_{n}(t)$ are easy to separate by windowing in the time domain as described in [19]. Finally, they can be Fourier transformed into frequency domain as

$$
H_{n}(f)=\mathcal{F}\left[h_{n}(t)\right]
$$

The magnitude plot of $H_{n}(f)$ in decibel units is the most common representation, since it shows the amount of distortion of all the harmonics as a function of frequency [1]. 


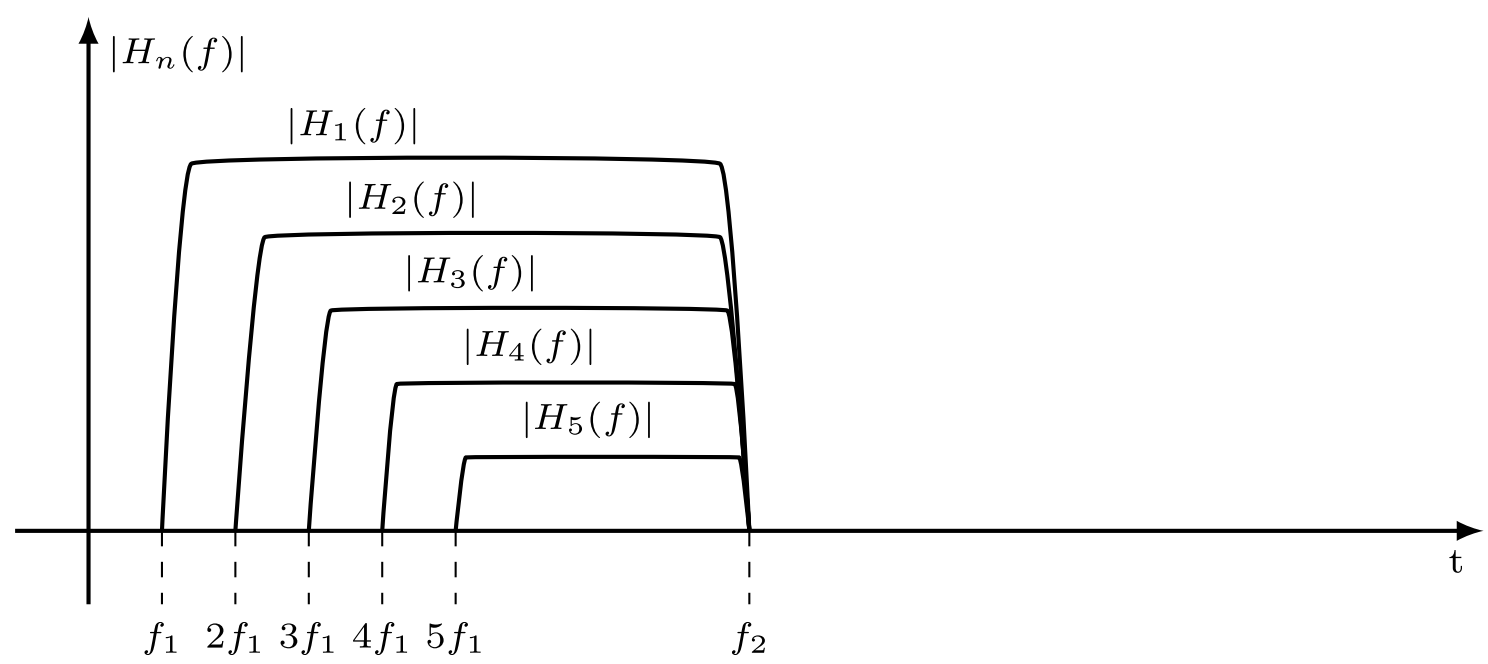

(a)

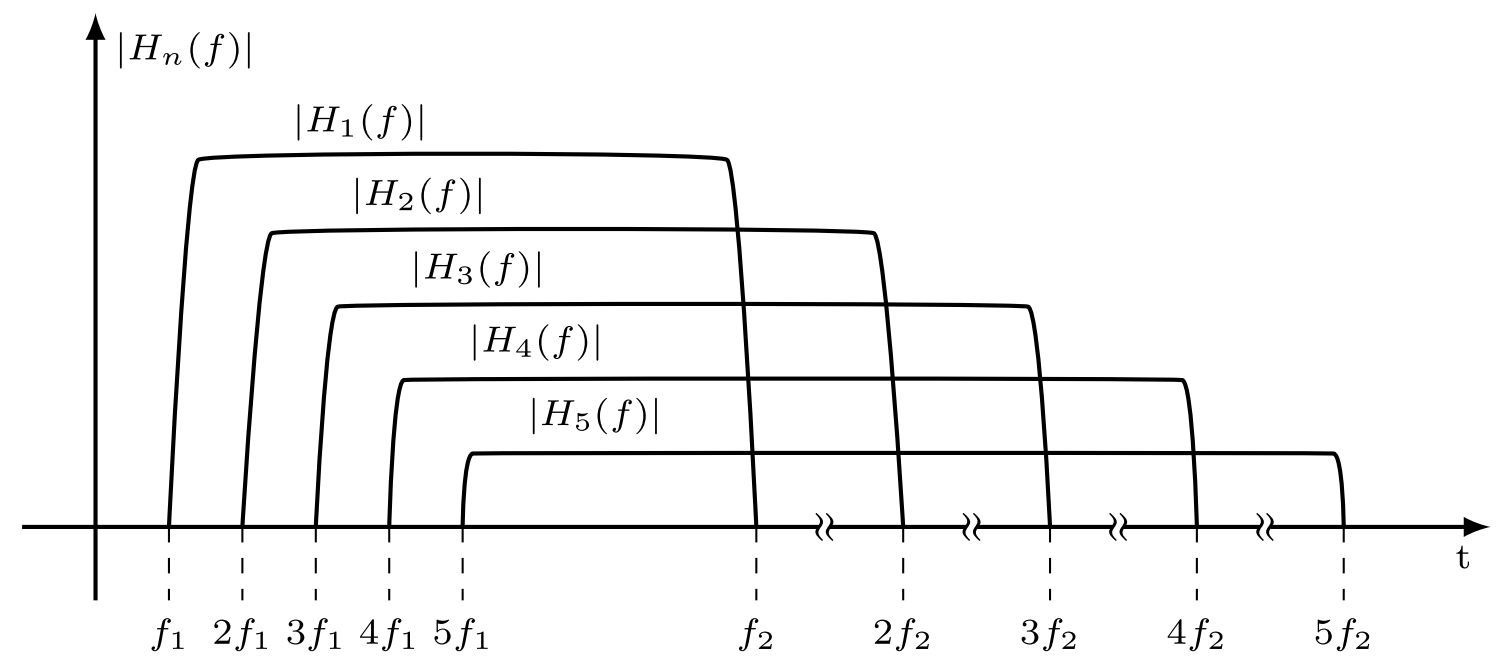

(b)

Figure 6. Schematic representation of magnitude spectra of HHFRs $H_{n}(f)$. (a) The deconvolution using Eqs. (4) or (7) set the upper bandwidth limit to $f_{2}$. (b) The deconvolution using the analytic expression in Eq. (38) does not limit the frequency bandwidth of HHFRs.

It is worth noting that using the usual way of deconvolving the impulse response using Eq. (4) or Eq. (7), the frequency bandwidth of HHFRs is $\left[n f_{1}, f_{2}\right]$, where $n$ is the number of studied HHFRs. Consequently, all the HHFRs stops at frequency $f_{2}$ as depicted in Fig. 6a.

On the other hand, if we use the deconvolution defined in Eq. (38) which is based on the analytical expression of the swept-sine signal in frequency domain, the frequency bandwidth of HHFRs is $\left[n f_{1}, n f_{2}\right]$ as depicted in Fig. $6 \mathrm{~b}$. In other words, the deconvolution using the analytical expression leads to much larger frequency band of HHFRs. 


\section{SUMMARY OF SYNCHRONIZED SWEPT-SINE METHOD}

Since we have discussed several ways of generating the exponential swept-sine signal as well as several ways of deconvolving the impulse response, we shortly summarize, in this section, the main equations for the generation and the deconvolution that will be used as a theoretical basis for the implementation provided in Sec. A.2.

The synchronized swept-sine is generated using

$$
x(t)=\sin \left[2 \pi f_{1} L \exp \left(\frac{t}{L}\right)\right] .
$$

where

$$
L=\frac{T}{\ln \left(\frac{f_{2}}{f_{1}}\right)},
$$

and where $f_{1}$ and $f_{2}$ are initial and final frequency respectively and $T$ is duration of the swept-sine. Contrary to previous works $[16,17]$ no added constraint is imposed on the rate of frequency increase $L$. However, if the initial phase is restricted te be zero at time $t=0$ the rate of frequency increase $L$ is defined as

$$
L=\frac{1}{f_{1}} \text { round }\left[\frac{f_{1}}{\ln \left(\frac{f_{2}}{f_{1}}\right)} \tilde{T}\right],
$$

$\tilde{T}$ being an approximative duration of the swept-sine. In such a case if the final frequency $f_{2}$ is chosen as an integer multiple of the initial frequency $f_{1}$, the synchronized swept-sine ends up with zero phase.

The deconvolution process is next performed in the frequency domain as

$$
\left.h(t)=\mathcal{F}^{-1}[\mathcal{F}[y(t)] \tilde{X}(f)]\right],
$$

where $y(t)$ is the acquired response of the nonlinear system under test to the synchronized swept-sine, and where the Fourier transform of inverse filter $\tilde{X}(f)$ is

$$
\tilde{X}(f)=2 \sqrt{\frac{f}{L}} \exp \left\{-j 2 \pi f L\left[1-\ln \left(\frac{f}{f_{1}}\right)\right]+j \frac{\pi}{4}\right\} .
$$

The impulse response $h(t)$ then consist of time-delayed higher harmonic impulse responses separated by time delays

$$
\Delta t_{n}=L \ln (n) .
$$

A full Matlab code, including all the necessary steps to implement the method is provided in appendix A.2.

\section{Illustrative Example}

In order to illustrate the advantage of using the synchronized swept-sine signal instead of the non-synchronized swept-sine signal for the estimation of the HHFRs, we present in this section the results of an experiment performed on a woofer (Pioneer W16FU90-51H). The woofer is excited using both the exponential (non-synchronized) sweptsine signal (1), called ESS, and the synchronized swept-sine signal (33), called SSS. The HHFRs of distorted current running through the woofer are estimated and compared with a measurement performed with a harmonic signal.

The measurement is performed using the sampling frequency $f_{s}=50 \mathrm{kHz}$. The parameters of the synchronized swept-sine signal are: the initial frequency $f_{1}=5 \mathrm{~Hz}$; the final frequency $f_{2}=500 \mathrm{~Hz}$; and the approximative time duration $\tilde{T}=10 \mathrm{~s}$. The amplitude of both excitation signals is $5 \mathrm{~V} \mathrm{rms,} \mathrm{high} \mathrm{enough} \mathrm{to} \mathrm{produce} \mathrm{distortion} \mathrm{artifacts}$ on the current. 

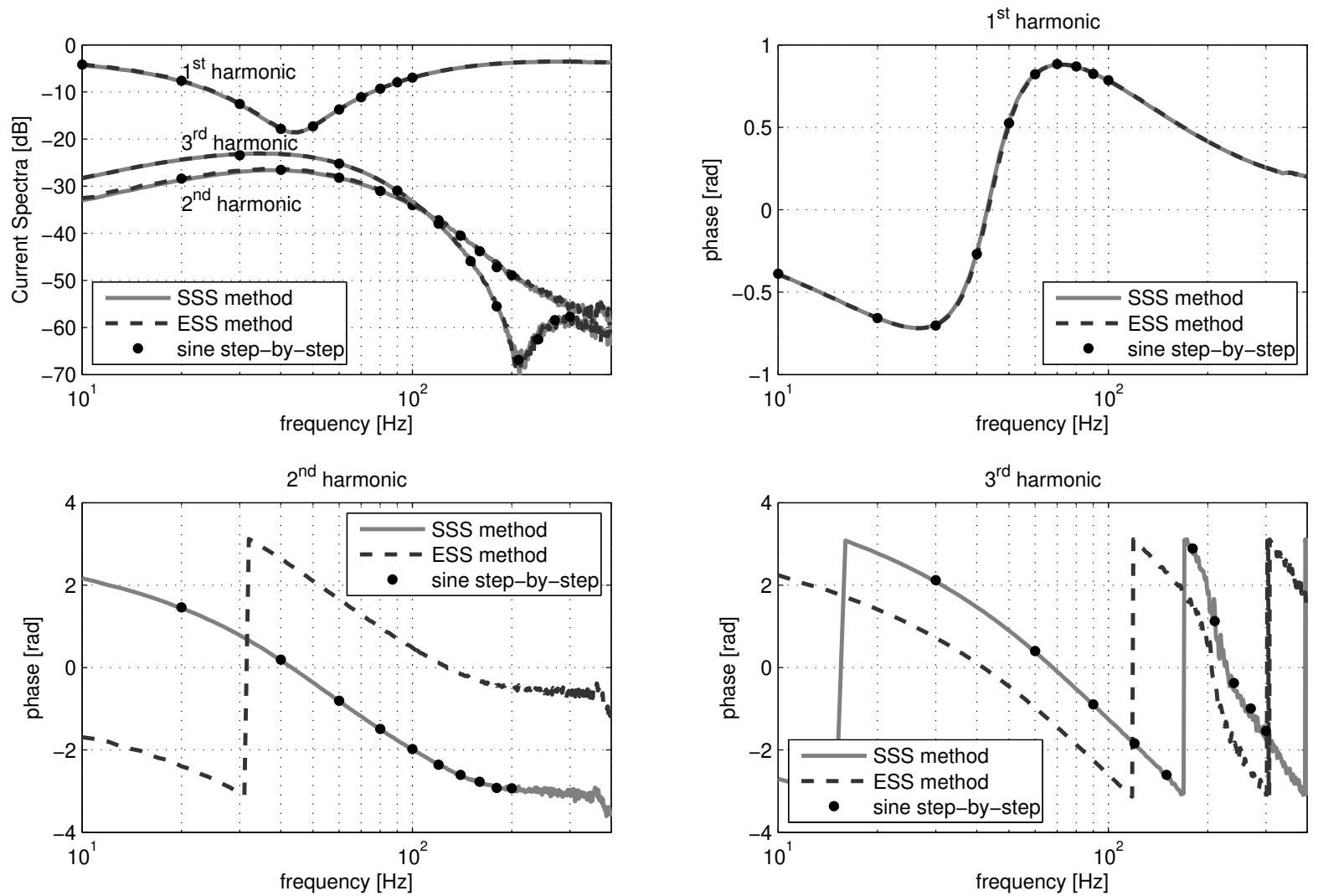

Figure 7. First three HHFRs of the distorted current passing through the woofer estimated using the non-synchronized swept-sine signal ESS (dashed line) and the synchronized swept-sine signal SSS (gray line), compared with a step-by-step measurement using a sine-wave excitation (dots).

The amplitudes and phases of the first three HHFRs estimated using both versions of exponential swept-sine signal are depicted in Fig. 7 and are compared with a measurement performed with a harmonic signal, the frequency of which is changed step-by-step from 10 to $100 \mathrm{~Hz}$ with a $10 \mathrm{~Hz}$ step. Whilst the amplitudes of all the HHFRs are correctly estimated for all the harmonics, the phases of the HHFRs $H_{n}(f)(n>1)$ are wrongly estimated in the case of the non-synchronized swept-sine signal (ESS). The reason for this error is the non-synchronization of the swept-sine signal as described in previous sections.

The harmonic phases presented in Fig. 7 are the phases of $h_{n}(t)$ related to the system under test and should be independent on the input signal $x(t)$. However, if the swept-sine signal is not correctly synchronized, the phases of $h_{n}(t)$ become dependent on the input signal $x(t)$ which is undesirable. On the other hand, if the swept-sine signal is correctly synchronized the phases of $h_{n}(t)$ remain independent on the input signal $x(t)$. This is confirmed in the illustrative example in Fig. 7, where the phases of all the HHFRs are correctly estimated in the case of the synchronized swept-sine (SSS).

\section{CONCLUSION}

In this paper, the theory of Synchronized Swept-Sine method has been revised. It has been shown that for a correct generation of the synchronized swept-sine a specific formula has to be used. Otherwise, the exponential swept-sine signal is not synchronized leading to an incorrect estimation of phases of Higher Harmonic Frequency Responses. If the synchronized swept-sine is generated using the equation derived in this paper the phases of Higher Harmonic 
Frequency Responses are correctly estimated.

Moreover, the analytical formula for the spectra of the synchronized swept-sine has been derived. Thus, the deconvolution of the impulse response can be made directly in the frequency domain using the analytical expression. Advantage of using the deconvolution with the analytical formula is that the estimated Higher Harmonic Frequency Responses have larger frequency bandwidth than when deconvolved the usual way. The use of analytical formula for the deconvolution is also less time consuming.

We have illustrated the advantages of using a synchronized swept-sine method over the usual non-synchronized one on analysis of current distortion of a woofer. Moreover, some application issues of the implementation are discussed in Appendix including a full Matlab code of the method.

\section{References}

[1] A. Farina, "Simultaneous measurement of impulse response and distortion with a swept-sine technique," in AES 108th convention, (Paris), Feb. 2000.

[2] A. Farina, A. Bellini, and E. Armelloni, "Non-linear convolution: A new approach for the auralization of distorting systems," in AES 110th convention, (Amsterdam), May 2001.

[3] P. G. Craven and M. A. Gerzon, "Practical adaptive room and loudspeaker equaliser for hi-fi use," in Audio Engineering Society Conference: UK 7th Conference: Digital Signal Processing (DSP), Audio Engineering Society, 1992.

[4] D. Griesinger, "Beyond mls-occupied hall measurement with fft techniques," in Audio Engineering Society Convention 101, Audio Engineering Society, 1996.

[5] J. O. Jungmann, R. Mazur, M. Kallinger, T. Mei, and A. Mertins, "Combined acoustic mimo channel crosstalk cancellation and room impulse response reshaping," Audio, Speech, and Language Processing, IEEE Transactions on, vol. 20, no. 6, pp. 1829-1842, 2012.

[6] D. G. Ćirić, M. Marković, M. Mijić, and D. Šumarac-Pavlović, "On the effects of nonlinearities in room impulse response measurements with exponential sweeps," Applied Acoustics, vol. 74, no. 3, pp. 375-382, 2013.

[7] P. Majdak, P. Balazs, and B. Laback, "Multiple exponential sweep method for fast measurement of head-related transfer functions," Journal of the Audio Engineering Society, vol. 55, no. 7/8, pp. 623-637, 2007.

[8] P. Dietrich, B. Masiero, and M. Vorländer, "On the optimization of the multiple exponential sweep method," Journal of the Audio Engineering Society, vol. 61, no. 3, pp. 113-124, 2013.

[9] A. Novak, M. Bentahar, V. Tournat, R. El Guerjouma, and L. Simon, "Nonlinear acoustic characterization of micro-damaged materials through higher harmonic resonance analysis," NDT $\mathscr{E}$ E International, vol. 45, no. 1, pp. 1-8, 2012.

[10] J. Legland, V. Tournat, O. Dazel, A. Novak, and V. Gusev, "Linear and nonlinear biot waves in a noncohesive granular medium slab: Transfer function, self-action, second harmonic generation," The Journal of the Acoustical Society of America, vol. 131, no. 6, pp. 4292-4303, 2012.

[11] G.-B. Stan, J.-J. Embrechts, and D. Archambeau, "Comparison of different impulse response measurement techniques," Journal of the Audio Engineering Society, vol. 50, no. 4, pp. 249-262, 2002.

[12] S. Bilbao and J. Parker, "A virtual model of spring reverberation," Audio, Speech, and Language Processing, IEEE Transactions on, vol. 18, no. 4, pp. 799-808, 2010. 
[13] J. Kemp and H. Primack, "Impulse response measurement of nonlinear systems: Properties of existing techniques and wide noise sequences," Journal of the Audio Engineering Society, vol. 59, no. 12, pp. 953-963, 2012.

[14] A. Benichoux, L. Simon, and R. Gribonval, "Convex regularizations for the simultaneous recording of room impulse responses," Signal Processing, IEEE Transactions on, vol. 62, no. 8, pp. 1976 - $1986,2014$.

[15] A. Novak, L. Simon, P. Lotton, and F. Kadlec, "A new method for identification of nonlinear systems using miso model with swept-sine technique: Application to loudspeaker analysis," in Audio Engineering Society Convention 124, Audio Engineering Society, May 2008.

[16] A. Novak, L. Simon, F. Kadlec, and P. Lotton, "Nonlinear system identification using exponential swept-sine signal," Instrumentation and Measurement, IEEE Transactions on, vol. 59, no. 8, pp. 2220-2229, 2010.

[17] M. Rébillat, R. Hennequin, É. Corteel, and B. F. Katz, "Identification of cascade of hammerstein models for the description of nonlinearities in vibrating devices," Journal of Sound and Vibration, vol. 330, no. 5, pp. 1018-1038, 2011.

[18] A. Novak, B. Maillou, P. Lotton, and L. Simon, "Nonparametric identification of nonlinear systems in series," Instrumentation and Measurement, IEEE Transactions on, vol. 63, no. 8, pp. 2044-2051, 2014.

[19] S. Müller and P. Massarani, "Transfer-function measurement with sweeps," Journal of the Audio Engineering Society, vol. 49, no. 6, pp. 443-471, 2001.

[20] L. Tronchin, "The emulation of nonlinear time-invariant audio systems with memory by means of volterra series," Journal of the Audio Engineering Society, vol. 60, no. 12, pp. 984-996, 2013.

[21] A. Farina, "Advancements in impulse response measurements by sine sweeps," in Audio Engineering Society Convention 122, Audio Engineering Society, May 2007.

[22] T. Erneux, Applied delay differential equations, vol. 3. Springer, 2009.

[23] B. Boashash, "Estimating and interpreting the instantaneous frequency of a signal. i. fundamentals," Proceedings of the IEEE, vol. 80, no. 4, pp. 520-538, 1992.

[24] L. Cohen, "Instantaneous frequency and group delay of a filtered signal," Journal of the Franklin Institute, vol. 337, no. 4, pp. 329-346, 2000.

[25] J. R. Carson and T. C. Fry, "Variable frequency electric circuit theory with application to the theory of frequency-modulation," Bell System Technical Journal, vol. 16, no. 4, pp. 513-540, 1937.

[26] C. E. Cook, Radar Signals: An Introduction to Theory and Application. Academic Press, 1967.

[27] M. Garai and P. Guidorzi, "Optimizing the exponential sine sweep (ess) signal for in situ measurements on noise barriers," in Euronoise 2015, EAA, Euronoise.

[28] A. V. Oppenheim, R. W. Schafer, J. R. Buck, et al., Discrete-time signal processing, vol. 2. Prentice-hall Englewood Cliffs, 1989.

[29] A. Torras-Rosell and F. Jacobsen, "A new interpretation of distortion artifacts in sweep measurements," Journal of the Audio Engineering Society, vol. 59, no. 5, pp. 283-289, 2011. 


\section{APPENDIX}

\section{A.1 Fourier Transform of the Synchronized Swept-Sine Signal}

The Fourier transform of the analytical signal $z(t)$ from Eq. (39) is defined as

$$
\begin{aligned}
Z(f) & =\int_{-\infty}^{\infty} z(t) \exp (-j 2 \pi f t) d t \\
& =\int_{-\infty}^{\infty} \exp \left\{j 2 \pi\left[L f_{1} \exp \left(\frac{t}{L}\right)-f t\right]\right\} d t
\end{aligned}
$$

which can be expressed as

$$
Z(f)=\int_{-\infty}^{\infty} \exp (j 2 \pi \Theta(t)) d t
$$

where

$$
\Theta(t)=L f_{1} \exp \left(\frac{t}{L}\right)-f t
$$

is the phase of the exponential term. The function $\Theta(t)$ is a convex function with a global minimum at

$$
t_{k}=L \ln \left(\frac{f}{f_{1}}\right) .
$$

Note, that this equation is the same as the one defining the group delay (45).

In order to find the Fourier transform defined in Eq. (A.1), we express the phase $\Theta(t)$ in a Taylor series around the time $t_{k}$ for each frequency $f$ as

$$
\Theta(t)=\sum_{n=0}^{\infty} \frac{\Theta^{(n)}\left(t_{k}\right)}{n !}\left(t-t_{k}\right)^{n} .
$$

We first solve all the derivatives $\Theta^{(n)}(t)$

$$
\begin{aligned}
\Theta(t) & =L f_{1} \exp \left(\frac{t}{L}\right)-f t, \\
\Theta^{\prime}(t) & =f_{1} \exp \left(\frac{t}{L}\right)-f, \\
\Theta^{(n)}(t) & =\frac{f_{1}}{L^{n-1}} \exp \left(\frac{t}{L}\right), \quad(n \geq 2)
\end{aligned}
$$

and then we solve them for $t_{k}$ from Eq. (A.5)

$$
\begin{aligned}
\Theta\left(t_{k}\right) & =L f-f L \ln \left(\frac{f}{f_{1}}\right), \\
\Theta^{\prime}\left(t_{k}\right) & =f-f=0, \\
\Theta^{(n)}\left(t_{k}\right) & =\frac{f}{L^{n-1}}, \quad(n \geq 2) .
\end{aligned}
$$

The Taylor series from (A.6) can be then expressed as

$$
\Theta(t)=L f-f L \ln \left(\frac{f}{f_{1}}\right)+\sum_{n=2}^{\infty} \frac{f}{L^{(n-1)} n !}\left(t-t_{k}\right)^{n} .
$$


Putting the phase (A.13) to the Fourier transform (A.3) and using the principle of stationary phase [25, 26] allowing us to neglect the terms $n>2$ in (A.13) we can write

$$
\begin{aligned}
Z(f)= & \exp \left\{j 2 \pi f L\left[1-\ln \left(\frac{f}{f_{1}}\right)\right]\right\} \\
& \times \int_{-\infty}^{\infty} \exp \left[j \pi f \frac{\left(t-t_{k}\right)^{2}}{L}\right] d t .
\end{aligned}
$$

Substituting $u=t-t_{k}$ we get the function inside the integral even, leading to

$$
\begin{aligned}
Z(f)= & \exp \left\{j 2 \pi f L\left[1-\ln \left(\frac{f}{f_{1}}\right)\right]\right\} \\
& \times 2 \int_{0}^{\infty} \exp \left[j \pi f \frac{u^{2}}{L}\right] d u .
\end{aligned}
$$

The integral in Eq. (A.15) is one form of the Fresnel integral. Finally, solving the Fresnel integral we can write

$$
\begin{aligned}
Z(f)= & \exp \left\{j 2 \pi f L\left[1-\ln \left(\frac{f}{f_{1}}\right)\right]\right\} \\
& \times \sqrt{\frac{L}{f}} \exp \left(j \frac{\pi}{4}\right) .
\end{aligned}
$$

\section{A.2 IMPLEMENTATION AND APPLICATION}

The application and implementation issues of the proposed method are discussed in this section. A Matlab code is provided for each important step.

\section{A.2.1 Synchronized Swept-Sine Signal Generation}

The first step is the generation of the synchronized swept-sine signal. We first define the initial, final and sampling frequency and the time duration of the swept-sine signal.

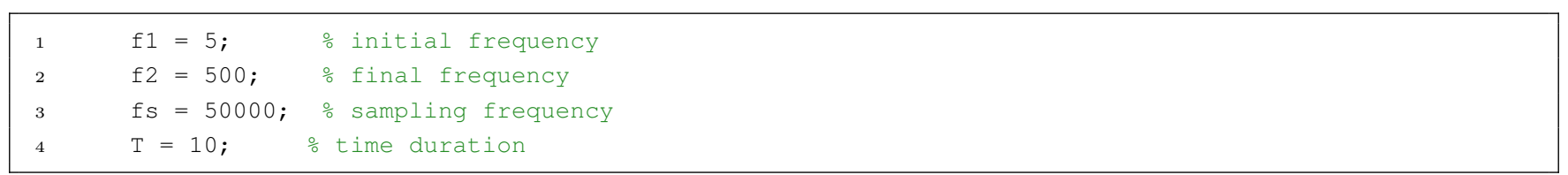

The choice of these parameters should be suited to the nonlinear system under test. In the example given in Sec. 7 we analyzed the current distortion of a woofer using an acquisition system with sampling frequency $f_{s}=50 \mathrm{kHz}$. Since the current distortion of a woofer is most important at very low frequencies we set the frequency range between $f_{1}=5 \mathrm{~Hz}$ and $f_{2}=500 \mathrm{~Hz}$.

Next, we generate the signal using Eqs. (47) and (49)

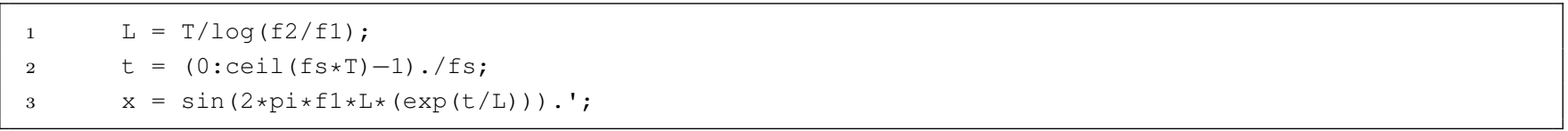

\section{A.2.2 Windowing, fade-in and fade-out}

For some applications it might be useful to apply a fade-in and/or fade-out window on the input signal [21, 27]. In [27], an optimal length and shape of these two windows is discussed. In the following code we propose a so-called raised cosine window [28], but any kind of window can be used [27]. 


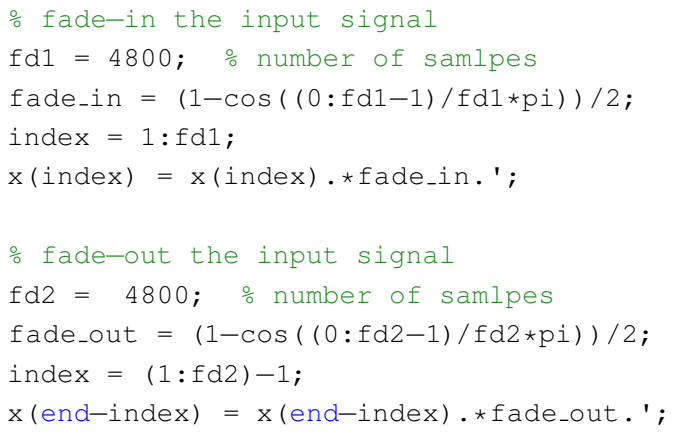

Note, that if we perform the deconvolution using (4) or (7) that applies the generated input signal data $x(t)$ to calculate the inverse filter, the fade-in fade-out windows are applied twice: once to the input signal $x(t)$ and once to the inverse filter. On the other hand, when the analytical formula for deconvolution (38) is used, the fading windows are applied only to the input signal $x(t)$.

\section{A.2.3 Output signal and its DC Component Filtration}

Once the synchronized swept sine signal is generated it can be used as the excitation to the nonlinear system under test. As discussed in [1], it is common to add a segment of silence after the excitation signal in order to avoid the cut of the tail of the systems response. In the following, we suppose the output signal $y(t)$ of the nonlinear system was correctly recorder and saved in Matlab under a variable y (a column).

It may happen that after recording the system response $y(t)$ the latter contains a DC component or even some very low-frequency content. This is either due to the nonlinear system under test itself [29], or due to the measurement setup, for example a vibrometer measuring a displacement signal may already be in a DC off-set. In both cases, the DC component should be filtered-out from the signal, otherwise it can contaminate the deconvolved impulse response with the scaled inverse filter $\tilde{x}(t)$ as shown below.

Let call the output signal with no DC component $y(t)$ and the DC component of the signal as $y_{D C}$ so the recorder signal may be in form of $y(t)+y_{D C}$. When convolving this signal with the inverse filter $\tilde{x}(t)$, it will result in

$$
\begin{aligned}
\left(y(t)+y_{D C}\right) * \tilde{x}(t) & =y(t) * \tilde{x}(t)+y_{D C} \cdot \tilde{x}(t) \\
& =h(t)+y_{D C} \cdot \tilde{x}(t) .
\end{aligned}
$$

Note, that the output of the deconvolution is the desired impulse response $h(t)$ plus an undesired inverse filter $\tilde{x}(t)$ scaled by the $y_{D C}$ factor.

Thus, the DC component must be filtered out either electrically (many of Digital-to-Analog Converters have a protective high-pass filter implemented on chip), or by doing it manually. Once the DC component filtered-out, the deconvolution of the impulse response can be carried out.

\section{A.2.4 Deconvolution}

The deconvolution in frequency domain is implemented using Eq. (50) and the analytical expression of the inverse filter in frequency domain from Eq. (51).

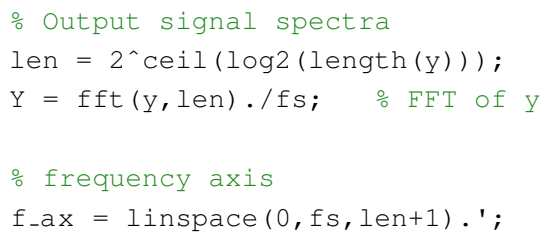




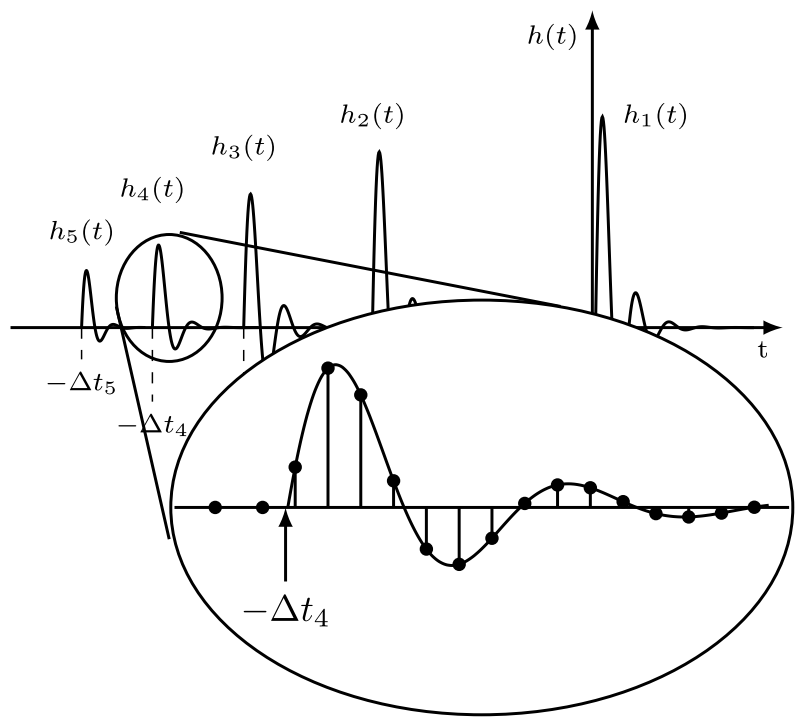

Figure 8. Schematic representation of time delay $\Delta t_{4}$ being between two samples of sampled impulse response $h(t)$.

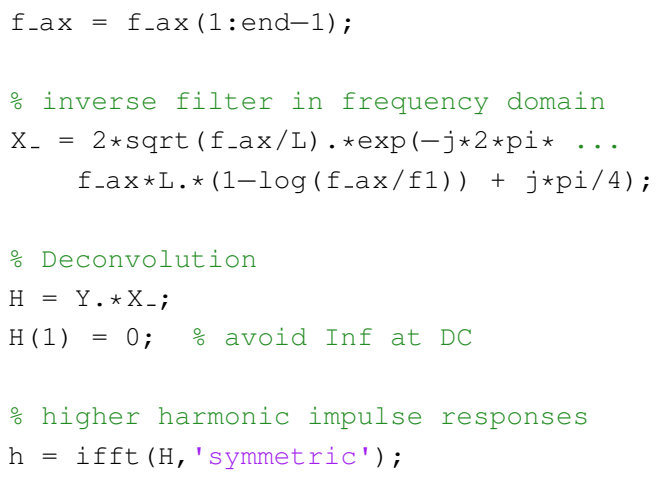

\section{A.2.5 Separation of Higher Harmonic Impulse Responses}

The deconvolution process results in the impulse response, that consists of time-delayed sum of higher harmonic impulse responses as depicted in Fig. 5. In order to separate them from each other, we must first calculate the time-moments $\Delta t_{n}$ in which these higher harmonic impulse responses appear using Eq. (52). In order to obtain directly the number of samples by which the higher harmonic impulse responses are shifted, we multiply the time delays $\Delta t_{n}$ by the sampling frequency $f_{s}$.

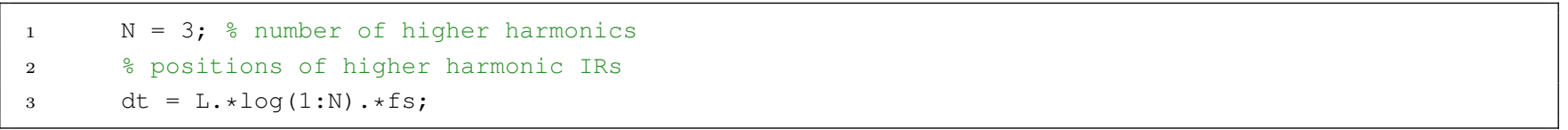

However, the number of samples dt defined as $\Delta t_{n} f_{s}$ is not necessarily an integer number leading to a non-integer sample shift to be realized. A schematic example of such a situation is depicted in Fig. 8. Thus, we first separate the higher harmonic impulse responses at the integer shift calculated as the rounded version of the exact non-integer number of samples and, after the separation, we implement the non-integer delay in frequency domain using the time shift property of the Fourier transform

$$
\mathcal{F}\left[f\left(t-t_{0}\right)\right]=\exp \left(-j \omega t_{0}\right) \mathcal{F}[f(t)]
$$


Finally, the HHFRs $H_{n}(f)$ are calculated as the Fourier transform of the higher harmonic impulse responses $h_{n}(t)$.

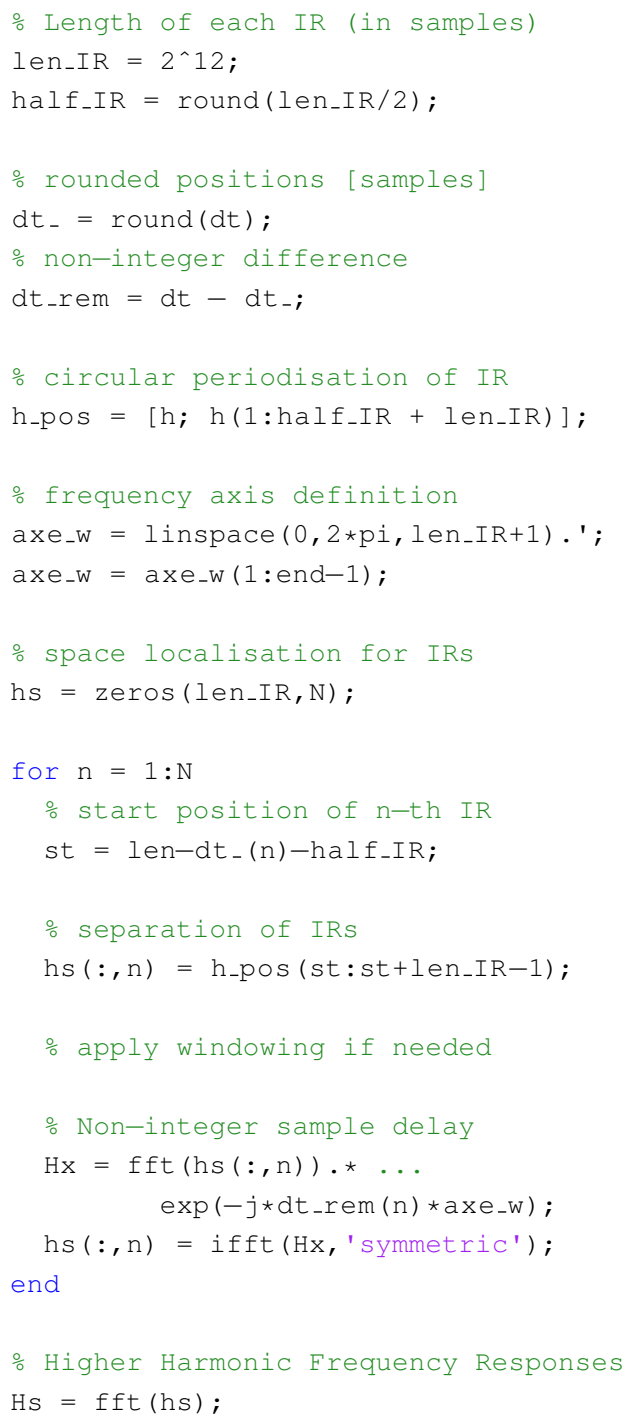

To smooth out the transitions between the impulse responses $h_{n}(t)$ and to control spectral ripples and time non-casual spreads, the impulse responses can be tapered prior to truncation using window functions such as the Hamming, the Bartlett, or the Kaizer windows [28]. 